\title{
Epidermic of Potts Disease at the Upper East Region of Ghana
}

\author{
Abdullah Yahaya, NiedowAlhassan, Abass Adam
}

\begin{abstract}
A retrospective study of potts diseases was conducted at the Tamale Teaching Hospital from January 2012 to December 2013. The highest number of patients was referred from Upper East Region (78\%). The disorder was common among women and young adults. Chronic back pain and neuropathy was the frequent presenting complains. The dorsal spine remains the site of preference while LIV2 recorded the highest incidence. The vertebral body was destroyed in all the cases and the fragmentary type of bone destruction was the common observation. The incidence of card compression demonstrated by CT was high. Other findings were paraspinal and epidural masses observed in and respectively (Chest Clinic, Tamale Teaching Hospital, and Tamale, Ghana)
\end{abstract}

Keywords: Computer tomography, Tamale Teaching Hospital, Upper East Region, Prevalence, Potts Disease, Epidemiology, Epidemy, Diagnoses, physiotherapy, WHO, MRI,Treatment, Evaluation, Chest Clinic, Paraphrases, Recovery, Chest Ward, Spinal Stenosis, Panological Fracture, Hypodens,Hypo-vitamin D, Calcification, Hypo-Calsenia, Differential Diagnose, anti-cocks and Dots Therapy.

\section{INTRODUCTION}
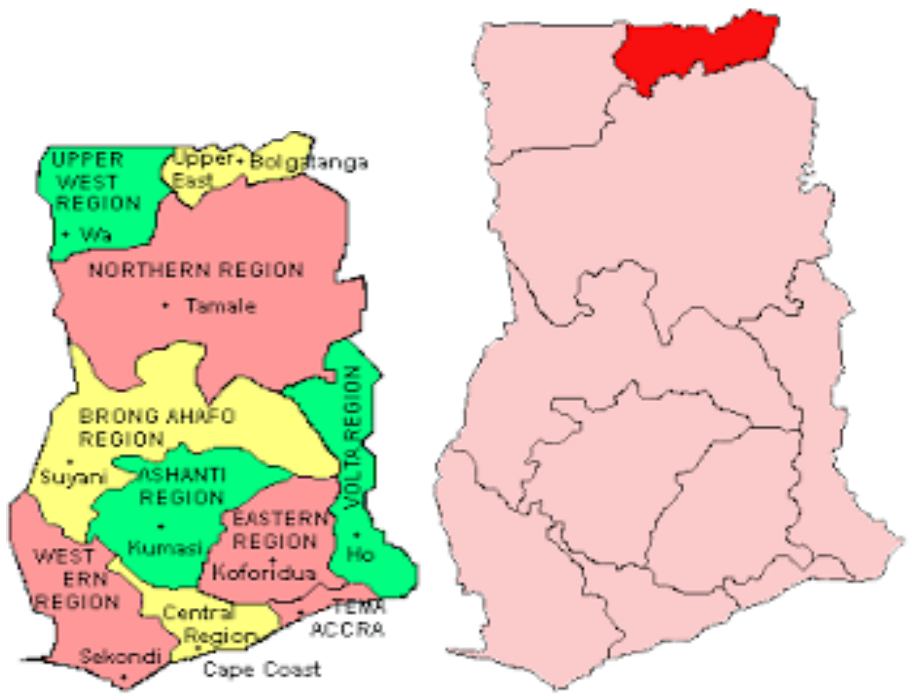

Map of Ghana showing upper east region

The Upper East Region is located in the north Ghana and is the second smallest of 10 administrative regions in Ghana, occupying a total land surface of 8,842 square kilometers or 2.7 percent of the total land area of Ghana, precisely located in the kingdom of Dagbon. The Upper East regional capital is Bolgatanga, sometimes referred to as Bolga. Other major towns in the region include Navrongo, Paga, Bawku and Zebilla.

\subsection{Location and Size}

The Upper East region is located in the north-eastern corner of Ghana, precisely located in the Kingdom of Dagbon, and bordered by Burkina Faso to the north and Togo to the east. It lies between longitude $0^{\circ}$ and $1^{\circ}$ West, and latitudes $10^{\circ} 30^{\prime} \mathrm{N}$ and $11^{\circ} \mathrm{N}$. The region shares boundaries with Burkina Faso to the north, Togo to the east, Upper West Region to the west, and the Northern Region to the south. The Upper East region is divided into 10 districts, each headed by a district chief executive. With only 21 per cent of the population living in urban areas, the region is the least urbanized in Ghana. In fact, together with the Upper West, they are two regions with a less than 20 per cent urban population. 
Ghanaian citizen by birth, childhood or parenthood constitute 92.5 percent of the population of the Upper East region. Naturalized Ghanaian citizen constitute 5.3 percent.

\subsection{Transportation}

Three National highways- N2, N10 and N11-and a few regional highways such as the R113, R114, R116 and R181, serve the region.

The N10 originates from Yemoransa in the Central Region and connects through Kumasi in the Ashanti Region and terminates at Paga in the Upper East Region. The national capital of Accra is also connected to the region by N2 which terminates in Kulungugu in the Upper East Region. Both these national routes are connected by the N11 which links the regional capital of Bolgatanga to Bimpiela, also in the region.

\subsection{Districts}

The political administration of the region is through the local government system. Under this administration system, the region is divided into 9 districts. Each District or Municipal Area, is administered by a chief executive, representing the central government but deriving authority from an Assembly headed by a presiding member elected from among the members themselves.

\subsection{Population}

The center of population of the Upper East Region is located in its capital of Bolgatanga. The population is primarily rural (79\%) and scattered in dispersed settlements. The rural population was 87.1percent in 1984 and $84.3 \%$ in 2000 .There was, thus, a 2.8 percent point reduction in the rural share of the population between 1984 and 2000 and a further 5.3 percent reduction between 2000 and 2010.

\section{BACKGROUND}

- Potts disease is rare in the UK but in developing countries it represents about $2 \%$ of cases of tuberculosis and 40 to $50 \%$ of musculoskeletal tuberculosis.

- Tuberculosis worldwide accounts for 1.7 billion infections, and two million deaths per year.

- Over $90 \%$ of tuberculosis occurs in poorer countries, but a global resurgence is affecting richer ones.

- India, China, Indonesia, Pakistan and Bangladesh have the largest number of cases but there has been a marked increase in the number of cases in the former Soviet Union and in sub-Saharan Africa in parallel with the spread of HIV.

- About two thirds of affected patients in developed countries are immigrants, as shown from both London and Paris and spinal tuberculosis may be quite a common presentation.

- The disease affects males more than females in a ratio of between 1.5 and 2:1. In the USA it affects mostly adults but in the countries where it is commonest it affects mostly

1. The onset is gradual

2. Back pain is localized

3. Fever, night sweats, anorexia and weight loss

4. Signs may include kyphosis (common) andlor a paravertebral swelling

5. Affected patients tend to assume a protective upright, stiff position

6. If there is neural involvement there will be neurological signs

7. A psoas abscess may present as a lump in the groin and resemble a hernia

i) A psoas abscess most often originates from a tuber culous abscess of the lumbar vertebra that tracks from the spine inside the sheath of the psoas muscle.

ii) Other causes include extension of renal sepsis and posterior perforation of the bowel

iii) There is a tender swelling below the inguinal ligament and they are usually apyrexial

iv) The condition may be confused with a femoral hernia or enlarged inguinal lymph nodes 


\section{INFECTION AND DISSEMINATION OF}

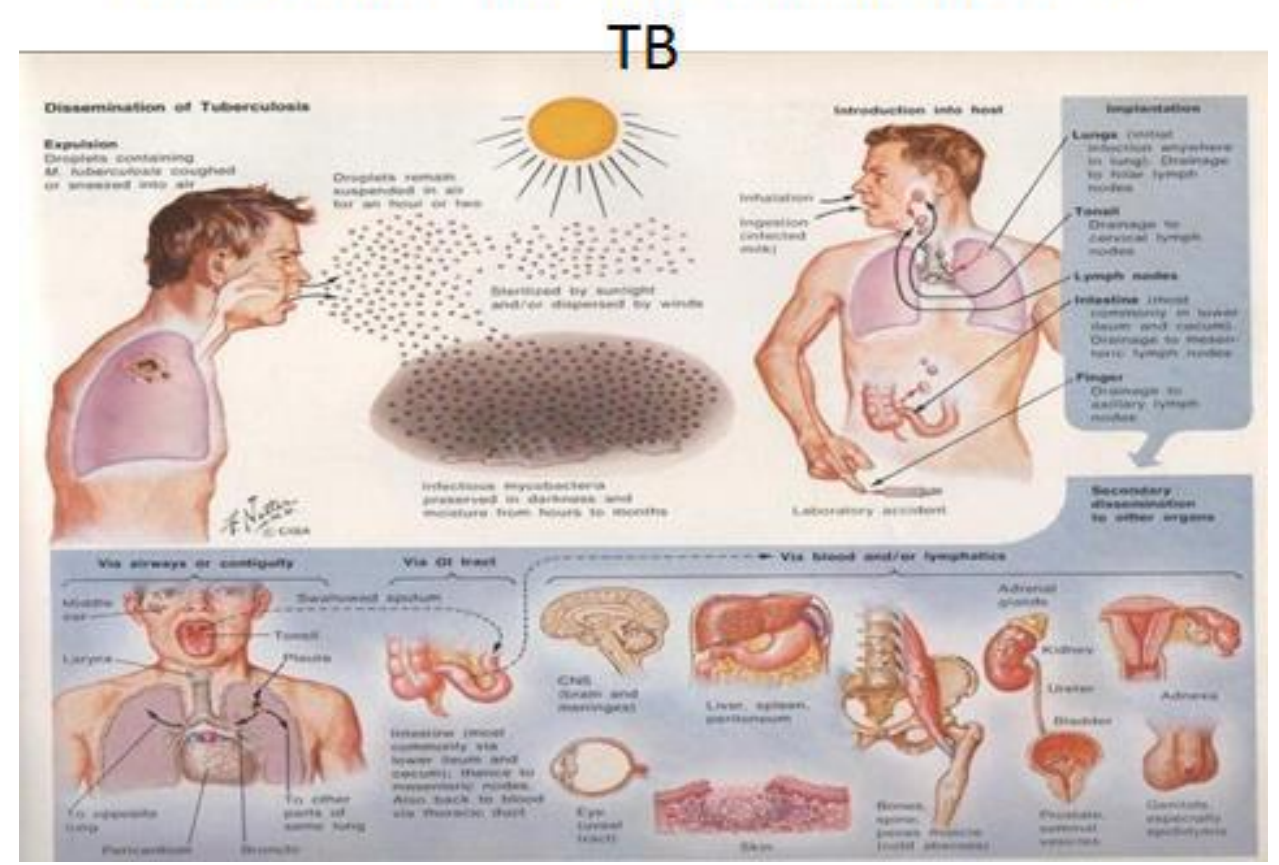

IJRMHS \& K.A.J. All rights reserved http://www.ijsk.org/ijrmhs.html

\section{LITERATURE REVIEW}

\subsection{Epidemiology}

Pott's disease is rare in the UK but in developing countries it represents about $2 \%$ of cases of tuberculosis and 40 to $50 \%$ of musculoskeletal tuberculosis.

Tuberculosis worldwide accounts for 1.7billion infections, and 2 million deaths per year.

Over $90 \%$ of tuberculosis occurs in poorer countries, but a global resurgence is affecting richer ones

India, China, Indonesia, Pakistan and Bangladesh have the largest number of cases but there has been a marked increase in the number of cases in the in the former Soviet Union and in sub-Saharan Africa in parallel with the spread of HIV

About two thirds of affected patients I developed countries are immigrants, as shown from both London and Paris and spinal tuberculosis may be quite a common presentation

The disease affects males more than females in a ratio of between 1.5 and 2:1. In the USA it affects mostly adults but in the other countries where it is commonest it affects mostly children

\section{MeThodology}

It is a descriptive report of the CT scans performed on 30 patients with proven Pott's disease from January 2012 to December 2013 at the Tamale Teaching Hospital in, Ghana.

Retrospective study of demographic records, patients of potts disease were contacted and interviewed. Clinical record books was reviewed

\section{After detailed explanation, legal permission was obtained from the patients}

\section{RESUltS}

Radiological investigations are central in the diagnosis and management of TB spine. In Ghana there is a dearth of literature on the disorder. This paper seeks to describe CT features of the bony and soft tissue changes in pott's disease. Retrospective survey revealed that there has been an increase in the number of pott'spatient'sreferred from upper east Region. The patients were important civil servants such as pott's disease is a spinal pathology as a result of of atrophy and collapse of the vertebral body.

Policemen, Teachers and students of higher learning institutions. It is therefore empirical to conduct further studies to explain the epidemics of pott's in the Upper East Region of the Northern Ghana 
Of late the treatment success of pott's disease at the Tamale Teaching Hospital has been recommendable. The method adopted is six months chemotherapy, four months physiotherapy and two months rehabilitation.

The disorder was common among women and young adults (76.7\%). Chronic back pain and neuropathy were the frequent presenting complains $(53.3 \%)$. The dorsal spine remains the site of preference while L1/L2 recorded the highest incidence (73.3\%). The vertebral body was destroyed in all the cases and the fragmentary type of bone destruction was the common observation. The incidence of cord compression demonstrated by CT was high (73.3\%). Other findings were Para spinal and epidural masses observed in (66.7\%) and (73.3\%) respectively.

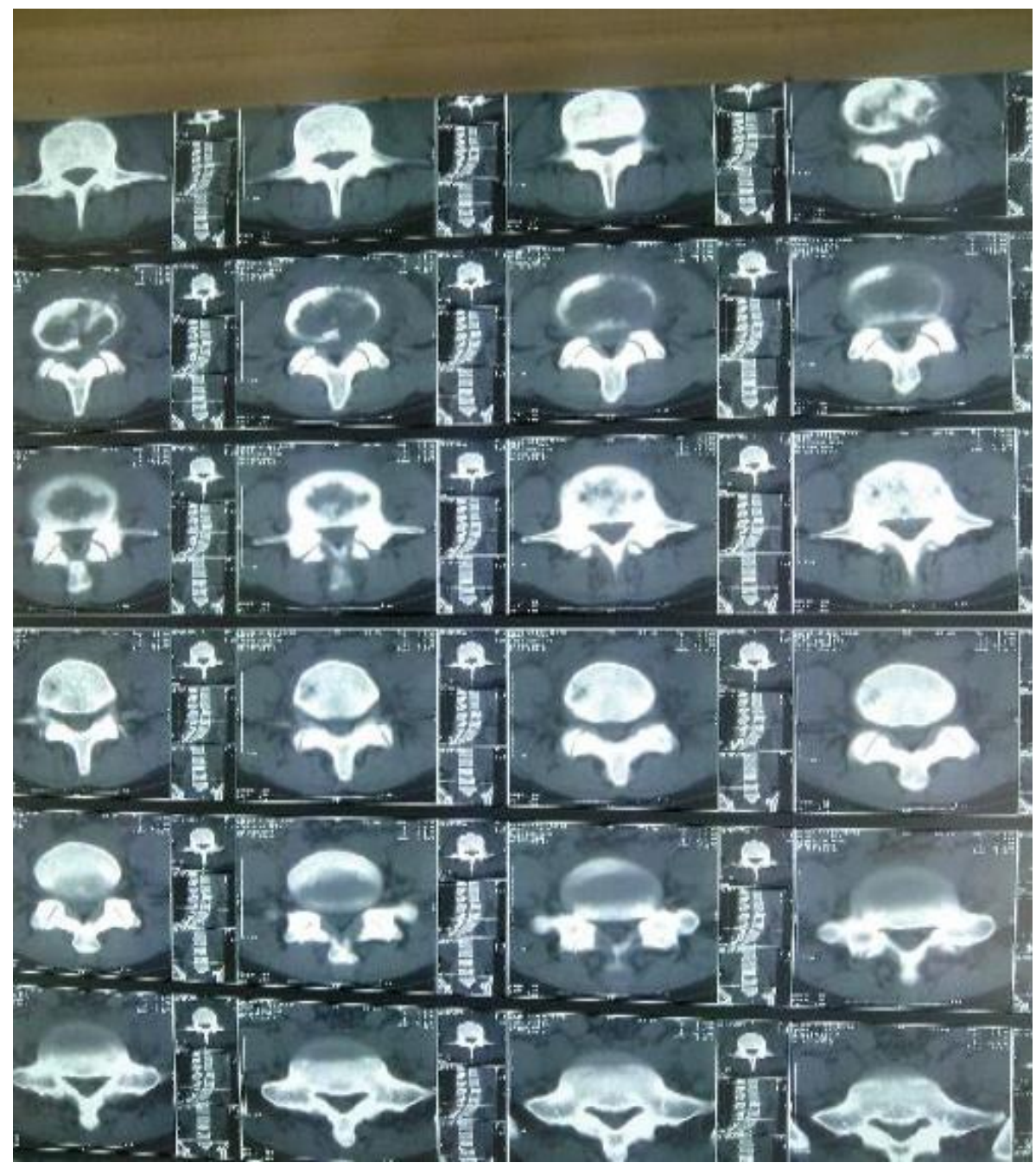

Computer tomography report of potts disease at TTH (Chest Clinic Register)

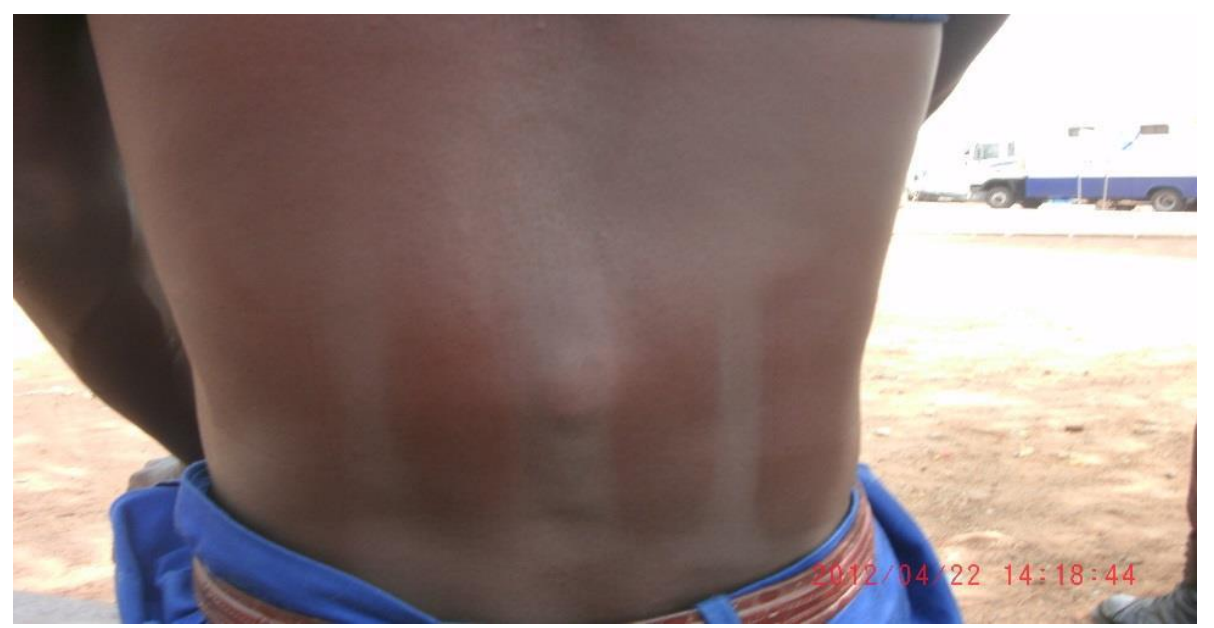

Ilopsoaabcess /L1/L2 degeneration (from Tamale Teaching Hospital Chest Clinic) by A. Yahaya 


\section{DISCUSSION}

Potts disease is extra pulmonary tuberculosis, which of late has increased in frequency at the chest clinic of the Tamale Teaching Hospital. As recorded by this research more than $70 \%$ of the confirmed cases were from the upper east regio0n of Ghana. Further studies will have to be conducted to explain this finding as documented in 2010 at the Teaching Hospital, it was found that delay in intervention after the incubation period, the mycobacteria disseminates to the spinal cord. If immunity compromises, the vertebral body gets hypotrophy, due to intoxication by the pathogen. The failure to absorb calcium, oxygen, vitamin c and vitamin will lead to spondylitis and finally to spinal collapse and spinal collapse causes stenosis which result in the manifestation of neurological deficit and to par paresis (3.). Also as proven in the results more women suffer pots disease than men. Further studies will have to be conducted to explain the phenomenon

\section{Conclusion}

CT images demonstrated the spinal level of destruction. Improved resolution also showed detailed changes within the outlined soft tissue masses, facilitating early diagnosis and prompt initiation of therapy. More than $70 \%$ of the cases were from the upper east region of Ghana.

Women and young adults were most vulnerable (76.7\%). Chronic back pain and neuropathy were the presenting complains $(53.3 \%)$. Further investigation is recommended to explain the epidemiology

\section{REFERENCES}

[1] Aquah et al (220120.The prevalence of tuberculosis at Northern Ghana; A Retrospective review at Tamale Teaching Hospital

[2] Obame et al. (-March 2000) Computed Tomographic Evaluation of Potts Disease In Accra

[3] Rockville Pike, Bethesda MD, National Center for Biotechnology Information, U.S. National Library of Medicine 860020894 USA

[4] Yahaya et al (2013). Effect of Delay in intervention on treatment outcome at the chest clinic of Tamale Teaching Hospital

[5] Aquah et al (220120.The prevalence of tuberculosis at Northern Ghana; A retrospective review at Tamale Teaching Hospital.

[6] Obame et al. (-March 2000) Computed Tomographic Evaluation of Pott's disease in Accra.

[7] Rockville Pike, Bethesda MD, National Center for Biotechnology Information, U.S National Library of Medicine 860020894 USA

[8] Yahaya et al (2013). Effect of delay in intervention on treatment outcome at the chest clinic of Tamale Teaching Hospital.

[9] Bhaskaram, P., Vijayakukumar. M. \&Hemalatha. P. (2010) Malnutrition and Childhood tuberculosis: report from National Institute of Nutrition, Indian Council of Medical Research Jamai Osmania, P.O. Hyerabad 500007,India.

[10] WHO (2000) The International Conference on Primary Health Care of 1978 at Alma-Ata Kazakhstan.

[11] WHO, author. (2003) Treatment of tuberculosis. Guidelines for National Programmes. $3^{\text {rd }}$ ed. Geneva: World Health Organization.

[12] WHO; 2005: Global tuberculosis control-surveillance, planning and financing: Russian Federation Geneva.

[13] Yahaya A., Acquah S, Ziem J and Sagoe K. (2013) Incidence of Pulmonary Tuberculosis in Northern Region. A retrospective survey of TB at Tamale Teaching Hospital. European American Journal of Scientific research.

\section{AUTHOR's BIOGRAPHY}

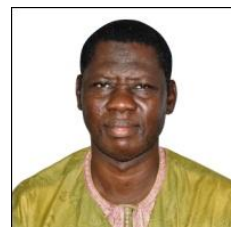

Dr Abdallah Iddrisu Yahaya, A Physician Consultant at Tamale Teaching Hospital, and also a Senior Lecturer in The Department of Internal Medicine of the School of Medicine and Health Sciences of the University for development Studies, Tamale. Ghana. 